\title{
Overview of the different processes of tungsten coating implemented into WEST tokamak
}

\author{
M. Firdaouss ${ }^{1}$, C. Desgranges ${ }^{1}$, C. Hernandez ${ }^{1}$, M. Richou ${ }^{1}$, H. Greuner ${ }^{2}$, B. Böswirth ${ }^{2}$, I. Zacharie- \\ Aubrun $^{3}$, T. Blay ${ }^{3}$, J. Bucalossi ${ }^{1}$, M. Missirlian ${ }^{1}$, F. Samaille ${ }^{1}$, E. Tsitrone ${ }^{1}$ \\ ${ }^{1}$ CEA, IRFM, F-13108 Saint-Paul-Lez-Durance, France \\ ${ }^{2}$ Max-Planck-Institut for Plasma Physics, Boltzmannstr. 2, D-85748 Garching, Germany \\ ${ }^{3}$ CEA, DEC/SA3C/LEMCI, F-13108 Saint-Paul-Lez-Durance, France
}

The Tore Supra tokamak is being transformed in an x-point divertor fusion device within the frame of the WEST (W-for tungsten-Environment in Steady-state Tokamak) project, launched in support to the ITER tungsten divertor strategy. The WEST project aims at testing W monoblock Plasma Facing Units (PFU) under long plasma discharge, with thermal loads of the same magnitude as those expected for ITER. The others Plasma Facing Components (PFC) will also be modified and coated with W to transform Tore Supra into a fully metallic environment. Different coating techniques have been selected, taking into account the specifications of the various PFC: heat loads, complex geometries (length up to 1m) and different substrates (CuCrZr for actively cooled PFC, graphite and CFC for other components). This paper gives an overview on the different processes used and the associated validation program and concludes on the adequacy of the $\mathrm{W}$ coating with the WEST experimental program requirements.

Keywords: coatings, plasma facing components, tungsten

\section{Introduction}

The development of tokamak plasma facing components (PFC) technology towards the fusion power plant goes through the use of metals as plasma facing material, and in particular tungsten (W), instead of the carbon material used in the past. The main objective of the WEST (W Environment in Steady-state Tokamak) project is to manufacture and test an ITER-like actively cooled tungsten divertor in a tokamak environment, in order to mitigate the risks for ITER. WEST provides a full tungsten environment. Besides the ITER like actively cooled plasma facing units (PFU) to be tested at WEST lower divertor, other WEST PFC make use of W coatings, as this is well adapted for less heavily loaded areas (lower heat and particle loads, hence erosion rate). W coatings also allow using existing carbon PFC to decrease the costs and the risks associated with the design and the manufacturing of new components.

\section{WEST context}

The upgrade of Tore Supra into WEST requires the modification of the previous plasma facing material from CFC to tungsten (see Figure 1). The plasma magnetic configuration is also modified, from the previous limiter configuration to a divertor configuration. The goal is to test the ITER W monoblock technology in realistic conditions at the lower divertor [1]. However, due to the longer time required for the manufacturing of these components compared to the others, WEST will start with a mix of ITER like actively cooled PFU and inertial (i.e. adiabatically loaded) PFU made of $\mathrm{W}$-coated graphite components [2].

Other actively cooled PFCs are newly designed and manufactured. The baffle [3], close to the lower divertor, aims at increasing the pumping efficiency. The upper divertor [4] is symmetric to the lower divertor and will allow upper and double null configurations. The ripple/VDE protections protect the top of the machine from the ripple losses and during the Vertical Displacement Events (VDE). These three types of PFC are made of W-coated copper alloy CuCrZr.

The last type of PFC in WEST consists of the reuse of some components of Tore Supra: 6 inner bumpers, 1 outer bumper and 10 antennae protections for the 5 antennae. They are all made of CFC N11 tiles either brazed (antennae protections) or bolted (bumpers) on a CuCrZr actively cooled support.

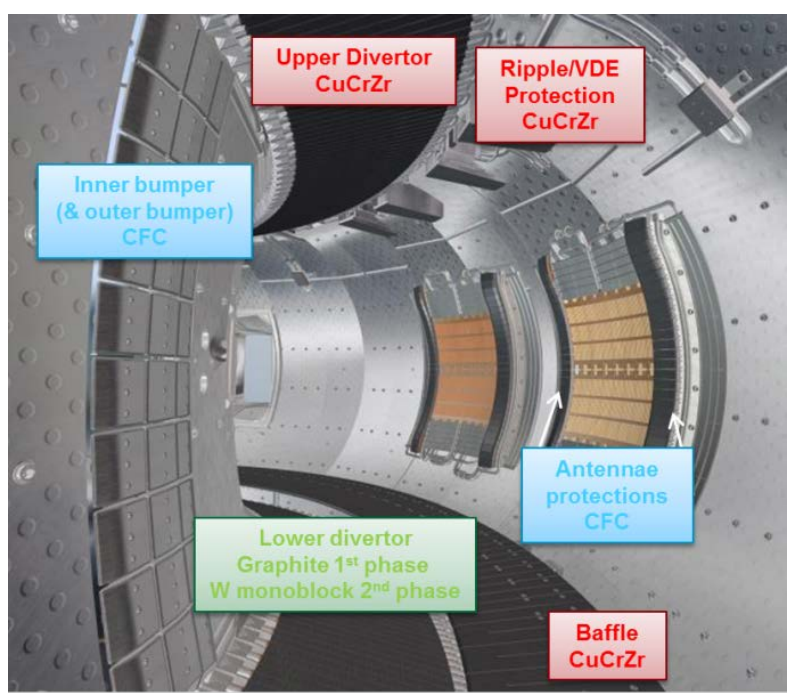

Figure 1: View of the in-vessel W-coated components in WEST configuration, including location of the different substrates (CFC, CuCrZr, graphite).

These three different types of PFC will have to be covered by a layer of $\mathrm{W}$ in order to provide a full $\mathrm{W}$ environment. The first part of the paper will present the main common specifications and their associated controls for the three kinds of coatings. Then each of them will be described in a dedicated section. Finally, the paper concludes on the adequacy of these coatings 
with the WEST experimental program, and gives perspectives on the development to be pursued.

\section{Common specifications and associated controls}

For all the coatings presented in this paper, the development has consisted in three successive phases. At first a R\&D phase was performed, that started in 2012, in parallel with the design of the components. Then, from 2014, the coatings have been qualified on mock-ups. Finally, the manufacturing of the PFC has started in 2015 , and the coatings at the beginning of 2016. The coated PFCs have then been delivered at CEA/IRFM, and their assembly started mid-2016 for a restart of WEST by end 2016.

During the R\&D phase, the main optimization parameter was the coating thickness. Indeed, it plays a key role for the lifetime of the component as well as to avoid delamination under thermal loading. The interface stress is lower with a low thickness (and thus the adherence and the resistance to delamination is better), but at the same time, low thickness leads to lower lifetime of the component due to erosion under plasma exposure. Interface stress depends also from the coating technique. So a trade-off has to be chosen in order to handle the WEST requirements in terms of heat and particle flux, but also other specifications:

- Ability to cover complex topologies (chamfer, gaps, round edges...) homogeneously. This is checked by measuring the coating thickness on samples at different locations by Scanning Electron Microscope. The dispersion within a batch should be lower than $5 \mu \mathrm{m}$.

- Low impurity content (in particular carbon and oxygen) that should be lower than $5 \%$ at to be compatible with plasma operation. This content is measured on samples by glow discharges optical emission spectroscopy techniques (GDOES). This method has also been cross checked during R\&D phase with energy-dispersive X-ray (EDX) spectroscopy

- Density should be at least $90 \%$, to ensure adequate thermal and mechanical properties of the coating. It can be measured by imbibition of samples.

- Surface should be exempt of defaults like scratch, crack, inclusion, spots... This is checked during the reception phase at CEA/IRFM by a systematic control with grazing light. A database showing the initial surface state for each PFC has been established, in order to compare it to the surface state after operation.

\section{W on non-actively cooled graphite PFC}

The Physical Vapor Deposition with Combined Magnetron Sputtering and Ion Implantation (PVD CMSII) process was initially developed for the ITERlike wall at JET on CFC DMS 780 by the NILPRP laboratory [5, 6] in 2007. This technique has been then successfully applied at ASDEX Upgrade on graphite tiles (SGL R6710). Since 10 years, this coating has been largely characterized $[8,9,10,11]$, both by high heat flux testing and during tokamak operation.

The PVD CMSII technique involves simultaneous magnetron sputtering of a target and high energy ion bombardment of the coating during its growth. The sputtered material is then condensed onto the PFCs. An interlayer of Mo is also used with this technique, to mitigate the thermal expansion mismatch between $\mathrm{W}$ and the substrate.

The very good experience with this process is the reason why it has been chosen for WEST. Some additional R\&D has still been carried out [7], to assess the influence of coating thickness on the delamination. From this study, a thickness of $10-13 \mu \mathrm{m}$ of $\mathrm{W}$ and2-4 $\mu \mathrm{m}$ of Mo has been selected.

935 tiles have been coated (see Figure 2), of 14 different types with embedded diagnostics (Langmuir probes, thermocouples and fiber Bragg grating).

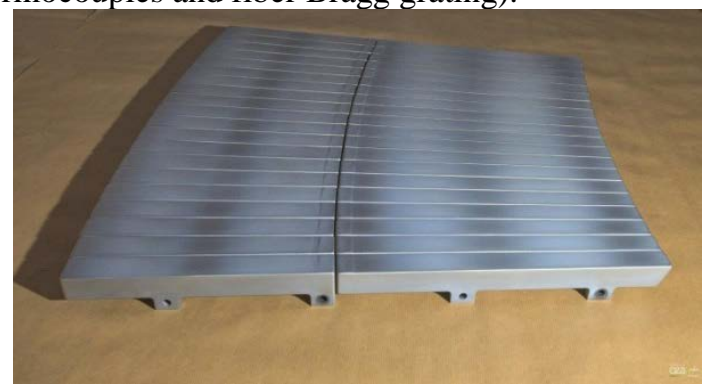

Figure 2: Start up graphite tiles for WEST lower divertor after W coating

High heat flux tests have been carried out on a full scale mock-up, in order to assess if the coating had performance similar to what is already described in the literature. The GLADIS facility [12] has been used to test this mock-up, with heat fluxes up to $8 \mathrm{MW} / \mathrm{m}^{2}$ and 5.6s pulse length, reaching a surface temperature of $1800^{\circ} \mathrm{C}$. A visual inspection of the coating has shown defaults near the most loaded area after few cycles (Figure 3)

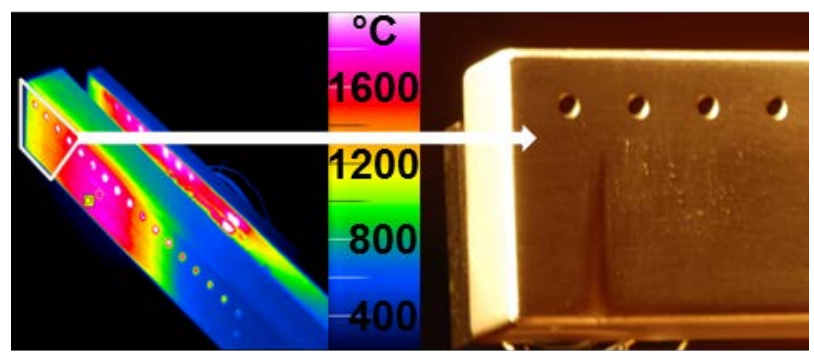

Figure 3: Temperature map during HHF test at $8 \mathrm{MW} / \mathrm{m}^{2}$ and defects on the surface

Subsequent FIB/SEM analysis of this area confirmed porosity formation at the interfaces [13], along with the carbidisation of the layers (MoC/WC/W2C) [14] as identified by STEM in previous studies [15]. The formation of tungsten carbide is a precursor of the coating delamination. 


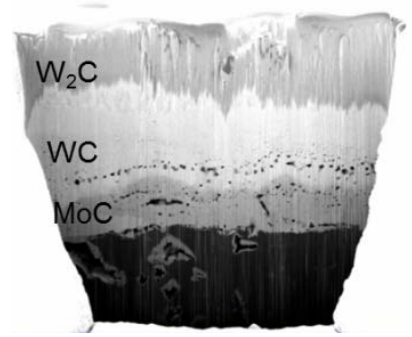

Figure 4: Carbidisation and porosity formation - FIB/SEM

Consequently the surface temperature should be limited to $1200^{\circ} \mathrm{C}$ during operation, with possible excursions up to $1800^{\circ} \mathrm{C}$ for a limited number of plasma discharges.

\section{W-PVD coating on actively cooled CuCrZr PFC}

Several challenging issues had to be faced for coating of these components, starting with the CuCrZr substrate, but also PFC with complex shapes including chamfers, round edges, holes [3, 4]. A R\&D program has been planned with the company DEPHIS, based on the Physical Vapor Deposition process.

The development was divided in several phases. The first phase enabled to assess the relevant coating thickness on small scale samples. Coating uniformity and thermal behavior has then been assessed under HHF tests. From this R\&D phase, a $15 \mu \mathrm{m}$ thickness has been selected [16]. 480 upper divertor PFCs, 150 baffle PFCs and 26 ripple/VDE protections have been coated with this process after manufacturing (Figure 5).

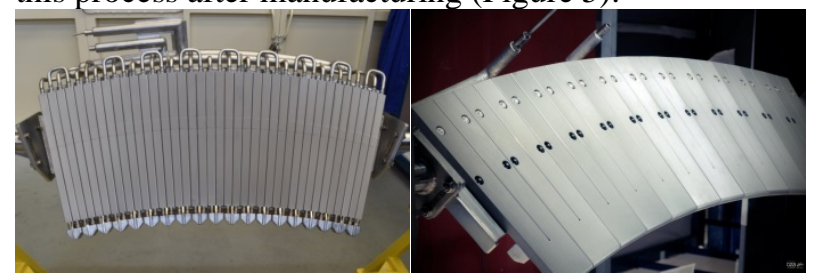

Figure 5: $30^{\circ}$ sectors after $\mathrm{W}$ coating: upper divertor (left and baffle (right)

High heat flux tests have been carried out on full size PFCs, to assess the performance of the $\mathrm{W}$ coating under the expected heat load during WEST operations. The GLADIS facility has also been used to qualify the components. The maximal heat flux was set to $10.5 \mathrm{MW} / \mathrm{m}^{2}$ for the upper divertor PFC, and $3.5 \mathrm{MW} / \mathrm{m}^{2}$ for the baffle PFC. In both cases, the maximal temperature was close to $500^{\circ} \mathrm{C}$ (Figure 6) which corresponds to the limit to maintain CuCrZr thermomechanical properties. Up to 500 cycles have been sustained by the baffle PFC. Visual inspection of the coating after the test has shown no delamination or default on the surface.

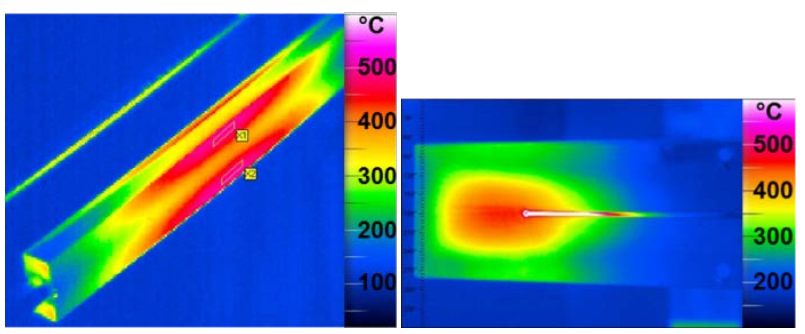

Figure 6: Temperature map during HHF tests: $10.5 \mathrm{MW} / \mathrm{m}^{2}$ on the upper divertor PFC (left) and $3.5 \mathrm{MW} / \mathrm{m}^{2}$ on the baffle PFC (right)

Consequently $\mathrm{W}$ coating on CuCrZr by PVD process is suitable for the expected heat flux on the actively cooled WEST PFCs.

\section{W-VPS coating on reused CFC PFCs}

The last type of PFC requiring a $\mathrm{W}$ coating in WEST is some of the already existing CFC N11 components. The 6 inner and the outer bumpers, composed of 212 individual CFC tiles $\left(\sim 10 \times 10 \times 3 \mathrm{~cm}^{3}\right)$ bolted onto a water cooled CuCrZr support, are reused. The 10 antennae protections, located on both sides of the 5 antennae used in WEST (2 Lower Hybrid (LH) launchers and 3 Ion Cyclotron Resonant Heating (ICRH) antennae), are also reused. They are made of CFC tiles that are brazed onto the water cooled support. Therefore it was not possible to dismantle the antennae protection for coating, whose length is around $1 \mathrm{~m}$.

Even though the individual bumper tiles are reused, there surface had to be machined, to adapt to the new plasma configuration. This machining solved the issue of cleaning the surface of the used tiles before coating. With the good results of the PVD CMSII on different carbon substrates (see above), it has been decided to apply the same process, with the same thickness, on the inner bumper tiles.

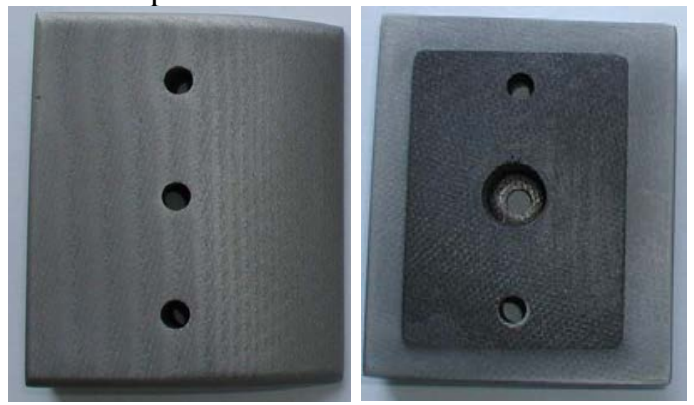

Figure 7: Inner bumper CFC tile with $\mathrm{W}$ coating: front and bottom face

However, this process is not applicable to the antennae protections, because of their size. Indeed, the maximal length acceptable is less than $40 \mathrm{~cm}$, due to the size of the PVD vessel. Therefore it has been decided to carry on a $\mathrm{R} \& \mathrm{D}$ program to develop $\mathrm{W}$ coating for large scale components. The Saint-Gobain company has been chosen for its past R\&D activities on developing $\mathrm{W}$ coating for JET [11].

Saint-Gobain applied a Vacuum Plasma Spraying (VPS) process. This process is performed in a controlled 
environment inside a sealed chamber, in a low pressure inert atmosphere, typically Argon. The material to be deposited is projected by a plasma torch that is mounted on a movable robotic arm. An interlayer of Mo is used with this technique.

The development was divided in different phases. The first phase enabled to assess the relevant thickness on sample for both Mo and W. From this R\&D phase, an $80 \mu \mathrm{m}$ thickness of $\mathrm{W}$ on top of an $80 \mu \mathrm{m}$ of Mo has been chosen. The coating thickness by VPS is higher than by PVD because of the process itself, while the density is slightly lower.

In addition to the 10 antennae protections, the 32 CFC tiles constituting the outer bumper were also coated with this process, to benefit from the higher $\mathrm{W}$ thickness: sputtering may be more important on the outer bumper than on the inner bumpers.

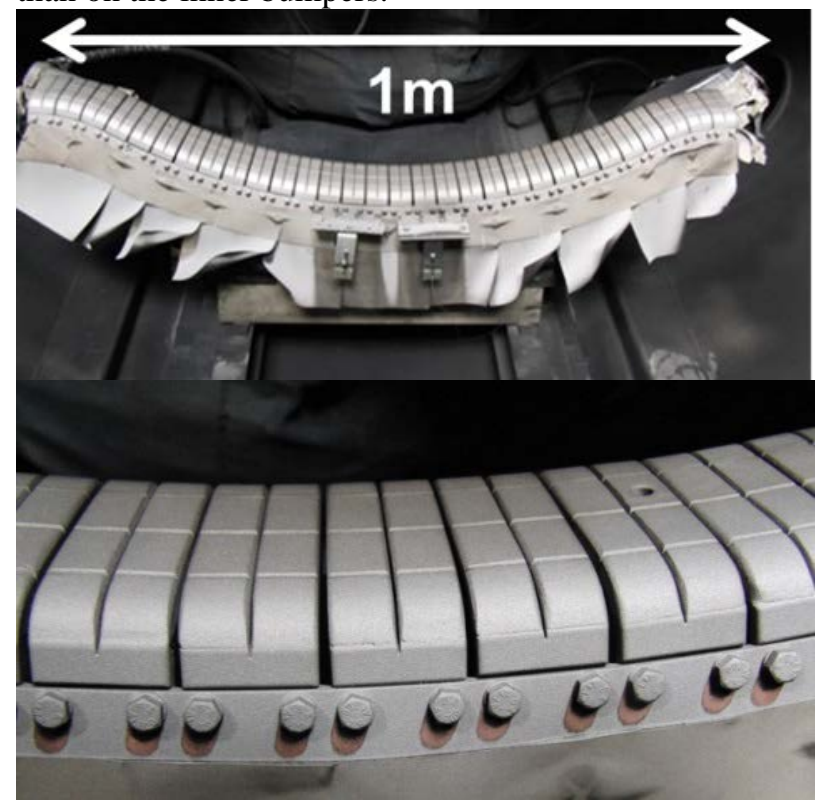

Figure 8: Antenna protection during the coating process (top) and close-up on the gaps and shaping (bottom)

High heat flux tests in GLADIS have been carried out on a mock-up of the antennae protection to validate the $\mathrm{W}$ coating process. The maximal applied heat flux of $8 \mathrm{MW} / \mathrm{m}^{2}$ resulted in a surface temperature of about $1000^{\circ} \mathrm{C}$ after $10 \mathrm{~s}$ loading close to steady-state. Since the initial screening at $3 \mathrm{MW} / \mathrm{m}^{2}$ before cycling, the mock-up has shown the two upper defects of the vertical edges shown on Figure 9. However, the most delaminated area (white arrow on Figure 9) did not grow significantly during the following 500 cycles. All defects occurred on the tile edges. Consequently all the edges of the antenna protections have been rounded by hand before coating.

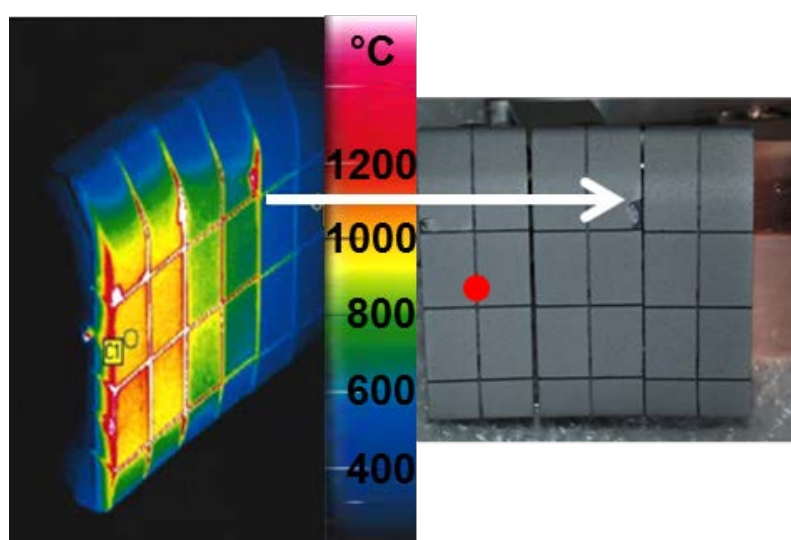

Figure 9: Temperature map during $8 \mathrm{MW} / \mathrm{m}^{2} \mathrm{HHF}$ loading on an antennae protection mock up. The right hand image shows the mock-up after cycling. The position of the beam center is marked with the red dot.

W coating by VPS process on re-used CFC components is suitable for the expected heat flux on the WEST PFCs.

\section{Conclusion}

The three $\mathrm{W}$ coating processes presented in this paper are all qualified by HHF tests to sustain the expected heat loads during WEST experiments. They also cope with the specifications like homogeneity (dispersion lower than 10\%), 90\% density and low impurity content required for plasma operations. The three types of substrates (graphite, CuCrZr and re-used CFC) are all compatible with $\mathrm{W}$ coating.

Several challenging issues have been solved during the $\mathrm{R} \& \mathrm{D}$, in particular handling complex shapes of the PFC or large scale components.

In addition to be a test bed for the ITER monoblock divertor, WEST may also serve as a test bed for new coating processes, with the use of dedicated PFCs. Indeed, WEST operation will allow assessing the performance of the different newly developed W coatings under tokamak environment (transient loads like ELMs, disruptions...) in particular for the actively cooled components. Effects of the roughness (due to the different substrates and the coating processes) on the erosion yield may also be evaluated.

$\mathrm{R} \& \mathrm{D}$ on healing processes for the different coatings developed for WEST is already on-going, to be able to repair possible damages on $\mathrm{W}$ coating after plasma operation.

\section{References}

1. J. Bucalossi et al., Fusion Engineering and Design 89 (2013) 907-912.

2. E. Tsitrone et al., these proceedings

3. T. Batal et al., 10.1016/j.fusengdes.2015.01.053 Fusion Engineering and Design 98-99 (2015) 1221-1225.

4. M. Richou et al., 10.1016/j.fusengdes.2014.12.017 Fusion Engineering and Design 98-99 (2015) 1394-1398

5. C. Ruset, et al., Phys. Scripta T128 (2007) 171-174.

6. C. Ruset et al., Fusion Engineering and Design, 84 (2009) 1662-1665

7. E Grigore et al., 10.1016/j.nme.2016.09.003, under 
publication

8. H. Maier et al., Journal of Nuclear Materials 363-365 (2007) 1246-1250

9. A Herrmann et al., 10.1088/0031-8949/2009/T138/014059, 2009 Phys. Scr. 2009014059

10. G.F. Matthews et al., Journal of Nuclear Materials 390-391 2009 934-937

11. R. Neu et al., Investigation of tungsten coatings on graphite and CFC, 10.1088/0031-8949/2007/T128/029,2007 Phys. Scr. 2007150

12. H. Greuner et al., Journal of Nuclear Materials 367-370 (2007) 1444

13. C Desgranges et al 2016 Phys. Scr. 2016014060

14. H. Maier et al., 10.1088/0031-8949/T167/1/014048, 2016

Phys. Scr. 2016014048

15. M. Rasinski et al., Thin Solid Films 531 21-24 (2013)

16. M. Richou et al., 2016 Phys. Scr. 2016 014029, 10.1088/0031-8949/T167/1/014029 